\section{DE LA ASTROARQUEOLOGÍA A LA ASTRONOMÍA CULTURAL} FROM ASTROARCHAEOLOGY TO CUL-
TURAL ASTRONOMY

\section{STANISLAW IWANISZEWSKI (*)}

\section{RESUMEN}

El autor bosqueja las relaciones entre el estudio de las prácticas calendáricas y astronómicas y el contexto sociocultural al que corresponden durante los últimos treinta años. Los diversos cambios experimentados pueden simbolizarse en los nombres de la astroarqueología. la astronomía megalítica, la arqueoastronomía, la etnoastronomía y la astronomía cultural. El autor describe, además, el estado actual de esta disciplina y proporciona una amplia bibliografía sobre el tema con fines de promover la investigación en España.

\section{ABSTRACT}

The author outlines a sketch of the development of the study of astronomical and calendrical practices in its sociocultural context during the last thirty years. This study has gone through many changes and these stages may be symbolized by the names of astroarchaeology, megalithic astronomy, archaeoastronomy, ethnoastronomy and cultural astronomy. The author describes this evolution, and the present state of this discipline and then gives a bibliography on the subject in order to promote such research in Spain.
\end{abstract}

(*) State Archeological Museum Poland. Dluga 52. 00950 Warsaw

El artículo fue remitido en su versión final el 23-III-94.
Palabras clave: Arqueoastronomía. Astronomía cultural. Etnoastronomía.

Key words: Archaeoastronomy. Cultural astronomy. Ethnoastronomy.

\section{INTRODUCCIÓN}

Aún hoy, casi 30 años después de las publicaciones de Gerald Hawkins (1963, 1964; Hawkins y White, 1965) sobre el significado astronómico del conjunto megalítico de Stonehenge, Inglaterra, el tema sigue siendo controvertido. Aunque el problema de Stonehenge ha sido gradualmente introducido en los libros de texto astronómicos y se convirtió, en cierto modo, en un tema clásico, los libros generales sobre la prehistoria europea ignoran esta materia. Esta actitud hacia la arqueoastronomía se puede atribuir, en parte, a su desorden metodológico y caos conceptual. Este artículo pretende cubrir dos objetivos: presentar el desarrollo de la arqueoastronomía y su estado actual y proporcionar una amplia bibliografía sobre el tema. Su combinación facilitará al lector los elementos necesarios para utilizar las ideas allí expuestas con prudencia. Por razones de espacio, no será posible exponer aquí su evolución en detalle (sobre todo en Mesoamérica, Perú y Suroeste en Américas, en Europa continental y otras partes del mundo), sino sólo sus líneas principales. 


\section{BRE VE HISTORIA DE LA ARQUEOAS- TRONOMÍA Y DE LA EVOLUCIÓN DE LOS ESTUDIOS SOBRE LAS ACTIVIDADES ASTRÓNOMICAS (Tabla 1)}

\section{Expansión (1963 - 1973/1975)}

Dos artículos y un libro de (ierald Hawkins (1963 y 1964: Hawkins y White. 1965) fueron decisivos para sacar a la lus el problema de los alineamientor astronomicos de los monumentos megaliticos en las Islas Británicas (1). El debate que se incio en la revista británica Antiquity (Atkinson. 1966: Hoyle, 1966b; Newham. 1966: Thom, 1966b; Hawkes, 1967 y otros) se limitó a los aspectos técnicos (2). Los primeros autores (Hawkins, 1968; Hoyle, 1966a,b; Newham, $1966 \mathrm{e}$ independientemente Charrière $\mathrm{y}$ Roos, 1964) se centraron en la metodología del trabajo de campo y en la asociación de los alineamientos de los monumentos megalíticos con los movimientos de los astros en el horizonte, apoyándose en los conceptos de Alexander Thom. Aunque Thom (1954 y 1955) publicó sus primeros artículos a mediados de los cincuenta, hasta 10 años más tarde sus estudios no se conocerían ampliamente. Su primer libro se editó en 1967 durante el debate sobre "Stonehenge decoded". Este libro, donde Thom reunió datos de más de 300 sitios megalíticos y presentó una investigación minuciosa enfocada a demostrar la alta precisión de sus alineamientos, iba a poner la discusión en otra dimensión. La posible existencia del factor astronómico en los alineamientos megalíticos, dado el número de sitios investigados y la aplicación de la estadística, simplemente no se pudo rechazar. Thom estableció cuatro líneas de investigación: 1) el estudio de la astronomía megalítica (1966b, más tarde puso mayor énfasis en las observaciones de los extremos lunares, 1971); 2) la investigación de la geometría de los monumentos megalíticos $(1961,1966 b) ; 3)$ la hipótesis sobre una

(1) Naturalmente, en el pasado existieron pioneros de la astroarqueologia, como Lockyer (1906), Somerville (1912) o Baschmakoff (1930).

(2) Esto se debió a que los primeros investigadores provenían de las ciencias exactas y de las disciplinas técnicas (astronomía, ingeniería, arquitectura) y en el debate utilizaban argumentos matemáticos que, al parecer, eran incomprensibles (véase, por ejemplo, Hawkes, 1967). Por otro lado, hay que recordar que el primer artículo de Hawkins (1963) salió a la luz un año antes de "Archaeology as Anthropology" de Binford y cinco años antes de la edición del "Analytical Archaeology" de Clarke. unidad de medida estándar. llamada "yarda megalitica" (1964): 4) la probabilidad de la existencia de un calendario de 16 meses (este tema, abandonado durante muchos años volvió a discutirse a finales de los ochenta). Thom insistió en la necesidad de lograr una gran precisión en el trabajo de campo y en la evaluación estadística de los alineamientos astronómicos. Aunque sus ideas sobre la "yarda megalítica" y el calendario fueron criticadas, la metodología empleada en su investigación fue generalmente aceptada y dio a la astroarqueología una impresión de madurez.

Los estudios de esta época se centraron en Stonehenge y otros monumentos megalíticos. Sus autores trataron de demostrar que los simples alineamientos de piedras se erigieron con una precisión asombrosa gracias a la cual se habían podido observar las salidas y puestas del sol y de la luna y calcular las fechas de los eclipses (aparte de los trabajos citados consúltense Colton y Martin, 1967; Anderson y Fletcher, 1968: Robinson, 1970; Hawkins, 1968; Atkinson, 1978; Thom, 1966a, 1971; Hawkins, 1965; Atkinson, 1974; MacKie, 1974). Dichos alineamientos fueron considerados como observatorios astronómicos (Hawkins, 1964; Hoyle, 1966a; Newham, 1966), que indicaban un cierto conocimiento astronómico, tratado como si fuera un saber científico en el sentido occidental. Las actividades astronómicas del Neolítico y de la Edad del Bronce (Brinckerhoff, 1976; Beach, 1977) se describían como "ciencia megalítica", "ciencia de la Edad de Piedra" y, sobre todo, como astronomía megalítica (Thom, 1966a, 1967: 92-106; Fleming, 1975; MacKie, 1977 a; etc.). Donde los alineamientos de los conjuntos megalíticos eran menos complejos y precisos se decía que sus constructores estaban menos desarrollados científicamente (Hawkins, 1965a: 130). A finales de este período MacKie (1974: 188-190) formuló su tesis sobre la presencia de una élite intelectual (los sacerdotes-astrónomos) que construyó observatorios megalíticos y manejó el saber científico durante el Neolítico y la Edad del Bronce.

Los estudios sobre la astronomía megalítica durante la década de los setenta se desarrollaron, en cierta manera, bajo la sombra de Alexander Thom y su familia (1972a,b, 1973, 1974a,b; Thom, Thom y Thom, 1974, 1975; Thom y Thom, 1978, Thom et alii, 1980) tanto en Bretaña como en las Islas Británicas y Stone- 
henge. Otros investigadores se propusieron revisar sus métodos visitando los sitios megalíticos, excavando algunos de ellos y repitiendo sus estudios (MacKic. 1974) o poniendo a prueba estadística sus resultados (Kendall, 1974). También se iniciaron los trabajos en el continente americano (Dow. 1967: Aveni, 1972) y los de Marshack (1964) sobre los sistemas de notación astronómica en el Paleolítico Superior que, posteriormente, recibirían mayor atención (Marshack. 1972).

La rigidez con la que se diseñaba el trabajo de campo. el uso de la trigonometría y de un lenguaje técnico fueron sólo alguno de los aspectos que obstaculizaban la entrada en este campo de los arqueólogos. Lo realmente perjudicial para la imagen de la astroarqueología era la falta de una teoría que tratara el saber astronómico en las sociedades prehistóricas (salvo MacKie, 1974). La idea de que las sociedades preliterarias fueron capaces de observar y registrar objetos astronómicos y predecir algunos de los fenómenos astronómicos era lo suficientemente atractiva para que varios astrónomos, matemáticos $o$ ingenieros abandonaran sus despachos y talleres para sufrir la incomodidad del arduo y laborioso trabajo de campo. La actitud de reserva e incluso de hostilidad (Atkinson, 1966; Hawkes, 1967), entre los arqueólogos, antropólogos e historiadores provocó un "desarrollo desequilibrado" de la astroarqueología: su parte técnica y relacionada con los métodos del trabajo de campo se estabilizó antes de que el debate teórico, arqueológico, antropológico o histórico hubiera sido iniciado. En esta situación, los astrónomos especularon, por ejemplo. sobre si el concepto de nodos, necesario para predecir eclipses en la ciencia moderna, pudo originar la noción de un dios potente ("tiene el poder" de eclipsar la luna y el sol) e invisible (ya que los nodos son sólo puntos imaginarios) de Isaac bíblico (Hoyle, 1966a: 256). La narración de Diódoro sobre un dios lunar que visitaba las islas boreales cada 19 años o sobre la isla en donde se veneraba al dios solar fue pronto identificada como una descripción de las observaciones del ciclo de nodos lunares (18.61 años) y con el monumento de Stonehenge (Hawkins, 1964: 1258; Hawkins y White, 1965). No extraña entonces que los antropólogos se alejaran de este campo de investigación.

Como resultado, a finales de la década de los sesenta aumentó el caos conceptual. La in- vestigación se limitó a la discusión técnica (sin duda muy importante), a la presentación de nuevos monumentos con orientaciones astronómicas o a la verificación de los estudiados por A. Thom. La "explosión" de nuevos datos y la precisión de los alineamientos megalíticos particulares dieron la impresión de una verdadera "caza de orientaciones".

Dos eventos señalaron una nueva etapa de la astroarqueología. En 1969 se celebró la primera reunión local sobre el tema, en la Universidad de Glasgow (MacKie, 1981) y. en 1972. el primer simposio de gran alcance, "El lugar de la astronomía en el mundo antiguo", con participantes procedentes de varios campos de la historia de la astronomía y de la astroarqueología, financiado por la Real Sociedad y la Academia Británica. Los artículos dedicados a los monumentos megalíticos (Atkinson, 1974; MacKie, 1974: Thom, 1974 y otros) discutieron la rigidez del trabajo de campo y la precisión de los alineamientos para las observaciones de eventos circumhorizontales. En el mismo volumen, Aaboe (1974) presentó un esquema descriptivo del desarrollo del saber astronómico en tres niveles y estableció una división conceptual entre la astronomía precientífica y científica. Lamentablemente este artículo quedó casi olvidado.

Por otro lado, la revista británica Journal for the History of Astronomy empezó a publicar artículos astroarqueológicos (sobre todo de Thom), lo que parecía demostrar cierta madurez y legitimidad de este nuevo campo de investigación.

\section{Consolidación (1973/1975 - 1981/1982)}

La publicación de Baity (1973) marcó un nuevo desarrollo conceptual. La autora empleó el término "arqueoastronomía" dándole un sentido más amplio que a los de "astroarqueología" o "astronomía megalítica" asociados con los monumentos megalíticos de Europa Occidental. También introdujo el concepto de "etnoastronomía" para denominar un campo interdisciplinario en el que se unían la astronomía, la etnología, la historia del arte y otros. En las Américas la "arqueoastronomía" pronto reemplazó a la "astroarqueología". Pero su estatus no estaba definido. Para Baity, eran subdisciplinas particulares, mientras que para los demás la arqueoastronomía se convertía en un campo interdisciplinario. 
El desarrollo de la propia arqueoastronomía y la propagación de su interdisciplinariedad se debe, sobre todo, a Anthony F. Aveni (1975). quien organizó dos conferencias internacionales en México D.F., en 1973 (junto con Horst Hartung), y en la Universidad Colgate, en 1975 (Aveni, 1977). Si bien la mayoría de los trabajos trataban de las actividades astronómicas de las altas culturas de Mesoamérica y Perú. la tercera conferencia internacional (Santa Fe, 1979) se interesó especialmente por las culturas noliterarias de Norteamérica (Williamson, 1981).

Mientras tanto, en Estados Unidos iniciaron sus estudios los astrónomos que se sintieron atraídos por el deseo de comprobar la hipótesis de Miller $(1955 a, b)$ sobre la presencia de la imagen de la supernova de 1054 d.C. en el arte rupestre norteamericano (Brandt et alii, 1975; Brandt y Williamson, 1977, 1979; Mayer, 1979). También la edición de "The Historical Supernovae" (Clark y Stephenson, 1977) despertó mucho interés ya que reunió los datos de las fuentes del Lejano Oriente sobre varias explosiones de novas y supernovas. Pareció que estos y otros datos podían usarse para verificar las estimaciones astronómicas acerca de la edad de la Nebulosa del Cangrejo (los restos de la supernova de 1054 d.C.) y de este modo contribuir al desarrollo de conceptos sobre la evolución estelar. Pronto se inició la búsqueda de relatos antiguos sobre otros fenómenos astronómicos (cometas, la estrella Sirio... Brecher, 1977) con fines de corregir el conocimiento astronómico actual. Este campo se definió como arqueoastrofísica.

Entre las décadas de los setenta y los ochenta en las Américas, la mayoría de los investigadores votó por el estatus interdisciplinario de la arqueoastronomía (y/o etnoastronomía). Estos estudios interdisciplinarios fueron considerados idóneos para la colaboración entre astrónomos y antropólogos (Eddy, 1981; Aveni, 1981a). Uno de los principales exponentes de este punto de vista, Anthony F. Aveni, siendo astrónomo, colaboró con Horst Hartung (historiador de la arquitectura mesoamericana), Tom Zuidema (antropólogo), Sharon Gibbs (historiadora de la astronomía) entre otros. Sus trabajos en Mesoamérica y en el Perú, publicados en inglés y español y en revistas arqueológicas, así como su libro (Aveni, 1980), preparado como un manual, dieron una enorme publicidad a la arqueoastronomía. Su posición recibió críti- cas cuando Edwin Krupp (1981: 56) observó que la interdisciplinariedad de la misma engendra el peligro de convertirla en un estudio científico superficial.

Otros científicos veían en la arqueoastronomía una prolongación de la historia de la astronomía. Mientras que ésta estudiaba la astronomía científica (en el sentido de Aaboe, 1974) aquélla debía ocuparse de la astronomía precientífica (Gibbs, 1979). Esta división de tareas investigadoras es precisa y clara, pero no define la metodología de la arqueoastronomía. Por eso Jane Young (1979: 14) propuso que fuera sólo una técnica de investigación cuyo objetivo fuera el de la historia de la astronomía. Así un científico era arqueoastrónomo cuando estaba en el campo e historiador de la ciencia cuando sacaba conclusiones.

Por su parte, John B. Carlson (1979), partidario del enfoque interdisciplinar, consideró que la arqueoastronomía (o la astroarqueología) en el sentido más restringido se limitaba a evaluar el saber astronómico a partir de la evidencia arqueológica no escrita, pero en el sentido más amplio, holístico, abarcaba la etnoastronomía y se refería al estudio del pasado y del presente.

La posición de Jonathan Reyman (1976, 1979) fue diferente: los estudios arqueoastronómicos deberían orientarse a resolver los problemas particulares de los sistemas sociales y no limitarse a demostrar ciertos alineamientos astronómicos. Insistiendo en definir los valores adaptativos de las actividades astronómicas antiguas, fue el primero en aplicar los conceptos cibernéticos al estudio arqueoastronómico. Según Reyman, la astronomía sirve como base para construir los calendarios que organizan y planean varias actividades sociales relacionadas con las estrategias de subsistencia y para hacer predicciones estrictas sobre ciertos fenómenos. Con estas dos funciones contribuye al mantenimiento de una población en un medio ambiente fluctuante e, incorporada a la religión, participa en la formación de un programa general adaptativo. Tomando ideas de Rappaport $(1971 \mathrm{a}, \mathrm{b})$, considera la religión como un sistema ideológico que genera el programa básico del sistema social para lograr su equilibrio con respecto al medio ambiente. En sociedades complejas o estratificadas, los sacerdotes son el vehículo que transmite este programa al resto de la sociedad y, al mismo tiempo, puede mani- 
pularlo. La función predictiva de la astronomía se presta a tales objetivos (Reyman. 1980).

En las Américas. aparte de Mesoamérica y el Perú, al menos dos áreas más fueron exploradas intensivamente: California y, sobre todo. el Suroeste norteamericano. En el importante artículo de Hudson y otros (1979), a partir del arte rupestre, se identificaron observatorios solsticiales potenciales en California (también en Hudson y Underhay, 1978; Hedges, 1981). Por su parte. Mayer (1979) buscó las imágenes de la supernova de 1054 en el arte rupestre. En el Suroeste, junto a estos mismos temas (WiIliamson et alii, 1977) se desarrollaron investigaciones etnoastronómicas (Farrer y Second, 1981; McCluskey, 1981; Young y Williamson, 1981).

Por su parte, Aveni (1981c) propuso el concepto de "arqueoastronomía tropical" para subrayar las diferencias existentes con respecto a la arqueoastronomía megalítica. Debido a la posición geográfica, en latitudes tropicales dominan los movimientos de los astros hacia arriba, encima, hacia abajo y por debajo, mientras que en latitudes altas prevalecen los movimientos hacia arriba, alrededor, y hacia abajo. Estas diferencias podrían tener como resultado diferentes cosmovisiones.

Cambios importantes sucedieron en los estudios megalíticos en Europa. Mientras Alexander Thom desarrollaba el paradigma de que las sociedades prehistóricas fueron capaces no sólo de observar sino también de registrar y predecir los fenómenos celestes, sin conocer la escritura, los arqueólogos cambiaban poco a poco su visión tradicional. En las Américas, los antropólogos pronto empezaron a participar en la investigación (p.e. Reyman, Hudson, Young, Broda), pero en Europa los arqueólogos tardaron más tiempo en reaccionar. Al comentar el desarrollo de la arqueoastronomía (astroarqueología) diez años después del "Stonehenge decoded", R.J.C. Atkinson (1975: 51) subrayó que reconocer la importancia de los estudios de A. Thom significaba cambiar la visión de las sociedades prehistóricas en Europa y que, ahora, a diferencia de su posición en la década de los sesenta, estaba dispuesto a ello.

Pronto se editaron en Inglaterra libros cuyos autores intentaban relacionar ciertos hechos arqueológicos con la existencia de un alto saber astronómico en el Neolítico y la Edad del Bronce (Burl, 1979a,b; MacKie, 1977a,b; Wood,
1978). Burl (1976) trató sobre todo de la arqueologia británica, incluyendo una descripción de la obra de Thom. sin intentar situar la astronomía dentro del marco sociocultural antiguo. A la lista de yacimientos megalíticos agregaba simplemente la mención de los alineamientos astronómicos. Escribiendo sobre Avebury discutió que la entrada a la tumba y la posición del muerto en su interior estuvieran orientadas astronomicamente. Hasta su tercer libro, no formula (Burl, 1979b) su tesis sobre una astronomía megalítica ceremonial, expresando su desconfianza hacia el concepto dominante de una astronomía megalítica científica. Burl negó también la existencia de la yarda megalítica definida como una unidad de medida uniforme. Considerando los círculos de piedra como monumentos funerarios concluyó que, posiblemente, la luna fuera el astro importante del culto a los muertos. A su vez MacKie (1977a,b) asoció el surgimiento del alto saber astronómico con la formación de una élite sacerdotal en las sociedades neolíticas. Los funcionarios del culto, especializados en el conocimiento geométrico y astronómico, los sacerdotes -astrónomos a tiempo completo-, se ocuparon de la conservación de ese saber. Partidario de esta hipótesis fue también Wood (1978) para quien la menor precisión de los monumentos megalíticos del Neolítico (zanjas y terraplenes), respecto a los de la Edad del Bronce (círculos e hileras de piedra), reflejaba la transición de una astronomía menos precisa, ceremonial, a otra especulativa, más precisa. Otros investigadores aceptaron también esa distinción, iniciándose el famoso debate sobre astronomía científica versus astronomía ritual (Ruggles y Whittle, 1981).

Los investigadores de aquel período estaban cada vez más convencidos de que debería emplearse la estadística para determinar si los alineamientos astronómicos eran casuales o no (Freeman y Elmore, 1979). Empezó a cambiar la opinión sobre la astronomía megalítica. Ya Heggie (1978: 456-457) observó que las orientaciones de los complejos megalíticos pudieron tener sentido en relación con actividades religiosas, rituales o simbólicas más que científicas o prácticas. El ya clásico artículo de Ellegård (1981) resumió el debate sobre astronomía ceremonial versus astronomía científica. A su vez, Thom (Thom, Thom y Burl, 1980) dio a conocer 
la mayor parte de sus datos sobre la precisión de los alineamientos solares y lunares.

En resumen, en las décadas de los setenta y ochenta se discutian los siguientes aspectos de la investigación:

1) El desarrollo de la estimación estadística. Para saber cuáles fueron las preferencias en la orientación de las sociedades neolíticas o de la Edad del Bronce era necesario encontrar un método objetivo que evaluara la intencionalidad de los alineamientos astronomicos. ('on ello se planteahan dos clases de estudios: el relativo a los propios alineamientos y a su evaluación (con la ayuda de excavaciones), así como el estudio de líneas visuales definidas a partir de formas en el horizonte que pudieron servir como puntos de referencia astronómica (Thom y Thom, 1980).

2) El problema de la intencionalidad en el grado de precisión de las orientaciones.

Uno de los primeros en detallar el método para el trabajo de campo fue Heggie (1981a,b). Los aspectos estadísticos fueron estudiados por Freeman (Freeman y Elmore, 1979; Freeman, 1982: Thom y Thom, 1982). Otros investigadores verificaron de nuevo los estudios de $\mathbf{A}$. Thom (Patrick, 1979; Ruggles, 1981, 1982a). En el debate (Moir et alii, 1980) se advirtió que los "observatorios lunares" que postuló no eran tan precisos como parecía. Por su parte, Burl (1980: 192) comentó que los antiguos orientaron los alineamientos hacia los eventos astronómicos circumhorizontales, no porque quisieran investigarlos sino porque tenían un sentido cosmológico (3) para ellos.

Nuevos simposios, congresos y reuniones de trabajo marcaron otra etapa en el desarrollo de la arqueoastronomía. A partir de 1979 se organiza un simposio sobre la arqueoastronomía y la etnoastronomía durante los Congresos Internacionales de los Americanistas. Además, se presentan sesiones durante las reuniones anuales de la Sociedad Astronómica Americana y también, a partir de 1980, en las de la Sociedad

(3) En la literatura etnológica y arqueológica se acostumbró usar el vocablo "cosmologia" para denotar los conceptos sobre el Universo entre las sociedades no literarias. Sin embargo, el uso moderno de la "cosmología" implica un conocimiento científico, en el sentido de la ciencia occidental. Es mejor usar la palabra "cosmovisión" para separar esta noción científica de la que no lo es (siempre y cuando se trate de la ciencia occidental). En esta ocasión, mantuve la palabra "cosmología" tal como la usó A. Burl.
Americana de Folklore. Se organizaron tres grandes simposios (Tabla 2).

Se crearon las primeras revistas especializadas como el Archaeoastronomy Bulletin (1977). posteriormente Archaeoastronomy. The Journal of the Center for Archaeoastronomy. Editado por John B. Carlson y Roy A. Williamson se convirtió en un cómodo vehículo para la transmisión de informaciones sobre el desarrollo de la arqueoastronomía en las Américas y, en menor grado. en el resto del mundo. Lamentablemente. la utilisima sección bibliográfica incluyó pocos articulos publicados en idiomas que no fueran inglés y español. En 1979 salió una segunda revista Archaeoastronomy. como suplemento al Journal for the History of Archaeoastronomy. Editada por Michael A. Hoskin, desde sus inicios publicó artículos sobre monumentos megalíticos y la arqueoastronomía americana.

\section{Ruptura (1981/1982 - 1986)}

En 1981 se celebró en Oxford la Primera Conferencia Internacional Sobre Arqueoastronomía (Aveni, 1982; Heggie, 1982a). Los participantes europeos presentaron una serie de trabajos relacionados con cuestiones metodológicas correspondientes al grado de precisión de la investigación y la validez de los conceptos estadísticos para confirmar alineamientos astronómicos. Los norteamericanos, aparte de las ponencias sobre ciertos alineamientos astronómicos de estructuras arquitectónicas particulares, en vez de limitarse al enfoque estadístico trataron de utilizar toda la evidencia antropológica (registros etnohistóricos, relaciones etnográficas incluso del siglo pasado), documentos escritos (códices e inscripciones prehispánicas en Mesoamérica), la evidencia arqueológica y la de la historia del arte para apoyar sus conclusiones. Se hizo patente que existían dos conceptos distintos sobre los estudios arqueoastronómicos.

En la primera parte de la década de los ochenta, se publicaron en Europa numerosas obras sobre el megalitismo. Heggie (1981a,b) reunió el material existente relacionado con los estudios sobre la astronomía y geometría megalíticas. Burl (1983) desarrolló su hipótesis sobre el uso simbólico de la astronomía en el contexto ritual asociado con las prácticas funerarias. Siguiendo la pauta establecida por Heggie, Ruggles $(1981,1982 a, b, 1983)$ reevaluó y sistematizó la metodología para investigar el punto de mira 
(hacksight) y la linea visual hacia el horizonte (foresight) ya que el análisis meticuloso de la obra de A. Thom pareció indicar que este investigador los empleaba con mucha subjetividad. Mas tarde, Ruggles (1984a) reunió datos sobre los yacimientos megalíticos en Escocia Occidental reevaluados y medidos de nuevo por su grupo. Esta investigación sentó unas bases sólidas para revisar los trabajos de Thom y. al publicar la lista de yacimientos con los dibujos del horizonte hacia donde se dirigían los puntos de mira. junto con el análisis detallado de sus acimutos, elevó el estándar de las publicaciones arqueoastronómicas. Thom, por su parte, siguió refinando sus evaluaciones de las orientaciones lunares (Thom y Thom, 1983).

Mientras tanto. en las Américas la discusión sobre el estatus de la arqueoastronomía entró en una nueva fase. Zeilik (1983: 5) fue uno de los primeros (el propio Zeilik cita a Reyman y Aveni) en reclamar la contextualización cultural de esta investigación que sin embargo, según él. no había madurado todavía lo suficiente para convertirse en una disciplina científica.

También se señaló que la insistencia en la interdisciplinariedad de la arqueoastronomía podía producir una falta de comprensión entre arqueólogos o etnólogos por un lado y astrónomos por otro (p.e. en Kehoe, 1981). Aparecieron importantes monografías etnoastronómicas (Urton, 1981; Chamberlain, 1982; Tedlock, 1982). En una de sus contribuciones, McCluskey (1981) reconstruyó el horizonte de observación de los hopi utilizando las fuentes etnográficas de la década de los treinta. Broda (1982) usó por primera vez en inglés el término español "cosmovisión" (visión estructurada del universo, véase Broda, 1978). Zeilik (1984a,b, 1985a, 1986) detalló las posibles estaciones de observación en el Suroeste, estableciendo los métodos de trabajo y resumiendo la información.

Aumentó el número de simposios y reuniones sobre el tema. A las reuniones celebradas anualmente por varias asociaciones científicas se añaden simposios regionales $y / o$ dedicados a un tema específico. En un período de cinco años se celebraron siete internacionales más uno nacional en Italia, donde antes no hubo mayores estudios (Tabla 2).

Puede concluirse que, entre 1981 y 1986 , las actitudes metodológicas en este campo podían reducirse a dos (Aveni, 1989b: 5): mientras que los investigadores americanos trataban de explicar las causas de las actividades astronómicas y las prácticas calendáricas en el contexto de las sociedades antiguas. los investigadores europeos (británicos) discutian su precisión.

\section{Nueva generación (1986 - 1990)}

Durante la Segunda Conferencia Internacional OXFORD 2 (Aveni, 1989a) sobre Arqueoastronomia celebrada en 1986 en Mérida. México. el primer enfoque fue llamado arqueoastronomía café y el segundo arqueoastronomía verde (Aveni, 1986, 1989b) por los colores del volumen dedicado al Nuevo (Aveni, 1982) y al Viejo Mundo (Heggie, 1982) respectivamente.

En Mérida, varios investigadores del Nuevo Mundo enfatizaron que los estudios arqueoastronómicos debían atender al contexto cultural. Gingerich (1989: 41) observó que, en el pasado, la arqueoastronomía se veía como una parte de la historia de la ciencia lo que condujo a considerar el conocimiento astronómico prehistórico como si fuera científico, y a evaluar entonces las culturas antiguas como si hubieran poseido una ciencia en el sentido occidental. Aveni (1989b) e Iwaniszewski (1989) situaron la arqueoastronomía en el contexto social. Carrasco (1989: 47) por su parte subrayó su estatus interdisciplinar y el papel de la astronomía en lo ritual, ceremonial y/o mítico. Algunos investigadores $(\mathrm{Ca}-$ rrasco, 1989; Iwaniszewski, 1989) volvieron a las ideas de Berthelot (1949), Eliade (1973) y Wheatley (1971) para ubicar las orientaciones astronómicas de los edificios en un contexto simbólico.

En la segunda mitad de la década de los años ochenta, un gran número de investigadores reconoce y adopta gradualmente el paradigma metodológico que sitúa las prácticas astronómicas en el contexto cultural. Aparecen las primeras publicaciones que, con el estudio del alineamiento, tratan de establecer su valor preciso e investigan las posibles visuales hacia el horizonte en su contexto, teniendo en cuenta el registro mítico, la evidencia arqueológica y la etnohistórica (Aveni et alii, 1988; Hedges, 1987). En este período nació el concepto de astronomía cultural (Iwaniszewski, 1990, 1991a, Tabla 1) considerada como una disciplina aparte.

Aparece también la corriente "revisionista". La nueva generación de investigadores cuestionó los resultados de los trabajos anteriores 
llegando a nuevas conclusiones (en Mesoamérica: Sprajc. 1987-88: en teoria astronómica: Schaefer, 1986). El dehate sobre la arqueoastronomia andina (Dearborn y Schreiber, 1986; Sadowski, 1989 y Ziółkowski. 1989) entró en una fase muy intensa y "caliente" (en Archaeoastronomy (JCA), 1987-88, 10: 22-34).

En Europa, después de la OXFORD 2. el paradigma de la arqueoastronomía verde desapareció (sobre todo la idéa de huscar alineamientos astronómicos muy precisos). Incluso sc pudo constatar (Rov. 1987: 24.5) que los "descubrimientos" de alineaciones en monumentos megalíticos que causaron la impresión del alto saber astronómico de las sociedades neolíticas. en muchos casos, se debían al propio investigador que conocía de antemano las orientaciones pertinentes y las "descubría" luego en el yacimiento. Por su parte, los arqueólogos al hablar de las orientaciones de ciertos monumentos megalíticos ignoraban totalmente el rigor metodológico tal como era propuesto, por ejemplo, por Ruggles (Midgley, 1985). En la teoría arqueológica se reconocía un simbolismo astronómico en las prácticas funerarias, en la localización y disposición espacial de los lugares ceremoniales, pero nada se decía sobre las observaciones astronómicas.

Aparecieron nuevas interpretaciones sobre el posible significado de los monumentos de Stonehenge (Burl, 1987; Roy, 1987).

Stonehenge I (ca. 3100 a.C.) se considera el lugar sagrado donde fueron depositados los restos simbólicos de los muertos (huesos calcinados, etc.). Para Burl fue un lugar de enterramientos colectivos como otros monumentos funerarios de tierra, madera o piedra ("túmulos", "tumbas megalíticas"); para Roy, una especie de locus sagradus donde se realizaban enterramientos simbólicos o dedicatorios. Estos ritos particulares relacionaban las comunidades de los vivos con el mundo de sus ancestros y con los individuos enterrados recientemente. La orientación astronómica solsticial tenía el propósito de coordinar los ciclos de los vivos con los del mundo de los muertos.

Stonehenge II (ca. 2150 a.C.) refleja un paso hacia lo ceremonial. En el lugar se celebraban rituales cuyas fechas coincidían con los solsticios de verano. Ya que los muertos no se enterraban dentro del recinto, se supone que se produjo un cambio de culto hacia una religión orientada al cielo. Stonehenge II pudo conver- tirse en el lugar donde los lideres y jefes de las comunidades locales se identificaban con los símbolos universales del poder religioso a través de ceremonias celebradas en fechas determinadas astronómicamente.

Stonehenge III (2000-1500 a.C.) marcó cambios nuevos. Los monumentos erigidos en el centro del recinto fueron utilizados por soberanos locales quienes efectuaban rituales anuales para renovar su posición social y legitimarla. La periodicidad de estos rituales dependió de los ciclos astronómicos.

A mbos enfoques no explicaban por qué Stonehenge continuó como un centro focal cuando los demás sitios neolíticos fueron abandonados.

Revivió la idea de A. Thom sobre la posible existencia de un calendario solar dividido en 16 meses (de 22 ó 23 días, Thom et alii, 1988; MacKie, 1988) y se estudiaron los antiguos calendarios a partir de varias fuentes etnohistóricas e incluso etnográficas (McCluskey, 1989).

En 1988-89 se organizaron los primeros simposios europeos sobre arqueoastronomía (Tabla 2, Interdisciplinarni Izsledvaniya, 17, 18; Rivista di Archeologia, Supplementi, 9). Pero el acontecimiento más importante para su desarrollo en Europa fue la organización de las reuniones semestrales "Astronomie et Sciences Humaines", la sucesiva edición de las memorias por Carlos Jaschek y Pierre Erny de la Universidad de Estrasburgo lo que significó practicamente la aparición de una revista nueva, Astronomie et Sciences Humaines a partir de 1988.

En general, en este período se inician los trabajos arqueoastronómicos en Europa continental, intensificándose los anteriores (p.e. sobre las orientaciones de los entierros, Barlai, 1980; Schlosser y Cierny, 1982; de iglesias, Guzsik, 1978; Firneis y Ladenbauer-Orel, 1978a,b; Köberl, 1984; de recintos rodeados con zanjas, Plešlova et alii, 1980).

\section{Transformación (1990 - 1993)}

La Tercera Conferencia Internacional OXFORD 3 sobre Arqueoastronomía reunió a los investigadores en Europa (en 1990, en Saint Andrews, Ruggles, 1993) y abordó problemas metodológicos. Mientras unos investigadores (Farrer, 1990) intentaron separar y dar una forma congruente a la subdisciplina de la etnoastronomía, denominada arqueoastronomía azul (por el color de la portada de los "abs- 
tracts" de la Primera (onferencia Mundial so. hre Finoastronomia organizada en 198.3 en Washington) otros (Ruggles y Saunders, e.p.) consideraron la arqueoastronomia como una subdisciplina de la astronomía cultural. La corriente "revisionista" americana se reveló con mayor fuerza. poniendo a prueba antiguas conclusiones (Köhler. 1991: Sprajć. 1993: Iwaniszewski. 199.3; Ziółkowski y Lebeuf. 199.3 y. sobre todo. la Mesa Redonda sobre el sistema ceque en Cuzco, organizada en 1992 en la Universidad de (olgate).

En las Islas Británicas, Ruggles (n.d.a.) se empeñó en desarrollar tres campos nuevos para la astronomía cultural: la verificación de las hipótesis astronómicas por una excavación sistemática de toda la región con la aplicación de los enfoques de la arqueología simbólica (la reconstrucción del paisaje simbólico): el uso de nuevos tipos de estadísticas (ya que las tradicionales no pueden aplicarse bien a los fenómenos sociales. Ruggles, 1990) y la aplicación del Geographic Information System en la investigación arqueoastronómica (Ruggles et alii, 1993). En Italia. Europa Central y Suroriental se multiplicaron las investigaciones y, lo que es más importante. se iniciaron trabajos donde la arqueoastronomía se incorpora (al fin) a la teoría arqueológica (p.e. Plešlova-Štiková, 1990).

En las Américas, Farrer y Williamson (1991) discutieron la metodología de la etnoastronomía. Aparecieron también publicaciones importantes que combinan la arqueoastronomía (líneas visuales) con la información arqueológica y etnohistórica y que, en cierto modo. constituyen un reto para la arqueología simbólica británica: se trata de las líneas de Nazca (Aveni. 1990) y de la reconstrucción del paisaje cultural del México Central (Aveni, 1991; Broda, 1991).

La astronomía cultural europea entró en una fase madura. Cada año se celebran reuniones sobre la arqueoastronomía europea en un país diferente (Tabla 2). Los más activos son ahora: el Reino Unido, Italia, Francia, Bulgaria, Alemania, Hungría y Polonia lo que provoca problemas adicionales, ya que aumenta considerablemente el número de trabajos publicados en diferentes idiomas. Por eso, salieron las primeras recopilaciones sobre la investigación arqueoastronómica en Italia, Bulgaria, Hungría y Polonia (Romano, 1992; Radoslavova, 1993; Pásztor, 1992; Iwaniszewski, 1992). Los esfuerzos (sobre todo de C. Jaschek) por crear una asociación cicntifica fructificaron en 1993 en Bulgaria. donde se organizi oficialmente la primera reunion anual de la Sociedad Europea para la Astronomia en la (ultura (SEAC). cuyo) primer presidente ha sido (live Ruggles de la Universidad de Leicester.

En 1991. para acelerar el proceso de intercambio de información John B. (arlson. David Dearborn y LeRoy Doggettse editaron también un nuevo boletín en Estados Unidos: Astronomy and Ethnoastronomy New's, The Quarterly Bulletin of the Center for Archaeoustronomy.

\section{¿Crisis? (1993 - 1996?)}

La Conferencia OXFORD 4, celebrada en Stara Zagora, Bulgaria produjo la impresión de que se había llegado a una fase crítica en el desarrollo de la astronomía cultural. A pesar de tratar de situar la investigación en el contexto sociocultural, fue clara la falta de teoría. Al parecer, las investigaciones han llegado a un punto de saturación y ya no pueden crearse nuevos enfoques capaces de generar modelos sobre el saber calendárico-astronómico en los sistemas culturales. Por otro lado, al mantener la visión de la interdisciplinariedad de la astronomía cultural se conservó la convicción de que los logros del trabajo proceden de la colaboración entre científicos y humanistas. Los próximos años nos darán a conocer cuál será el destino de la astronomía cultural.

\section{CONCLUSIONES}

Para los objetivos de este artículo presento las siguientes fases de desarrollo de la arqueoastronomía (Iwaniszewski, 1991b):

1) El período de recopilar, registrar y publicar datos. Dominan los estudios que describen las posibles orientaciones astronómicas de monumentos y complejos arquitectónicos.

2) La etapa de las interpretaciones calendárico-rituales. Aquí se trata de relacionar las orientaciones astronómicas con las prácticas ceremoniales de las sociedades antiguas o de describir los patrones de las distribuciones acimutales en categorías de fechas calendáricas importantes.

3) El período de las interpretaciones socioculturales. Abundan los trabajos que unen orientaciones particulares, observaciones de 
ciertos fenómenos astronómicos o los mismos acontecimientos astronómicos, con necesidades generadas por el sistema sociocultural tales como prácticas agrícolas, guerras, legitimación del poder de linajes particulares o de toda la clase elitista, creación de una cosmovisión dominante, etc.

4) Es lógico esperar el surgimiento de otra etapa, la de presentación de modelos generales sobre el papel de los fenómenos celestes en los sistemas culturales. Deberían aparecer estudios que relacionaran fenómenos celestes con elementos del medio ambiente natural, necesidades sociales y sicofisiológicas del hombre percibidas en el contexto cultural. El conocimiento de la teoría arqueológica, etnológica u otra es aquí indispensable.

Para terminar, resumiré mi opinión sobre la fase donde se encuentran las arqueoastronomías particulares a partir del esquema anterior.

California y Suroeste americano: entre la fase 2 y 3 .

Mesoamérica: culminando la fase 3.

Región andina: entre la fase 2 y 3.

Europa Occidental megalítica: aproximadamente en la fase 2.

Europa continental restante: apenas la fase 1, aunque parece, que en ciertas regiones (p.e. la Europa Central neolítica y eneolítica), se sitúa entre las fases 1 y 2.

Otras regiones: fuera de la clasificación ya que nunca se formaron núcleos de investigadores que de forma sistemática enfocaran sus estudios a una región particular o fase cronológica.

Es de esperar que, con el crecimiento de los estudios europeos, también en España aumentará el interés por impulsar la investigación arqueoastronómica.

\section{BIBLIOGRAFIA}

AABOE, A. (1974): «Scientific astronomy in Antiquity». En F. R. Hodson (ed.): 21-50.

ANDERSON, S.E. y FletChER, E.S. (1968): «The Inverse Stonehenge Problem». Current Anthropology, 9: 316318.

AtKInSON, R.J.C. (1966): «Moonshine on Stonehenge». Antiquity, 40: 212-216.

- (1974): «Neolithic science and technology». En F.R. Hodson (ed.): 123-131.

- (1975): «Megalithic astronomy: a prehistorian's commentw. Journal for the History of Astronomy, 6: 42-52.
AVFI. A.F (1972): "Astronomical tables intended for use in astro-archacological studiesw. American Antiquity, 37. 531.540

- (1975) (ed.): "Archaeoastronomy in Pre-Columbian America". University of Texas Press. Austin \& London.

- (1977) (ed.): "Native American Astronomy". University of Texas Press. Austin \& London.

- (1980): "Skywatchers of ancient Mexico". University of Texas Press. Austin.

- (1981)a: «Archaeoastronomy Today». En R.A. Williamson (ed.): 25-28.

- (1981)b: «Archaeoastronomy». En M.B. Schiffer (ed.): "Advances in archaeological method and theory". Academic Press. New York, 4: 1-77.

- (1981)c: "Tropical Astronomy". Science, 213, 4504: 161-171.

- (1982) (ed.): "Archaeoastronomy in the New World". Cambridge University Press. Cambridge.

- (1986): «Archaeoastronomy: past, present, and future». Sky and Telescope, 72, 5: 456-460.

- (1989)a (ed.): "World Archaeoastronomy". Cambridge University Press. Cambridge

- (1989)b: «Introduction: whither archaeoastronomy?n. En A.F. Aveni (ed.): 3-12.

- (1990)(ed.): "The lines of Nazca". American Philosophical Society. Philadelphia.

- (1991): «Mapping the Ritual Landscape: Debt Payment to Tlaloc During the Month of Atlcahualon. En D. Carrasco (ed.): "To Change Place. Aztec Ceremonial Landscapes". University Press of Colorado. Niwot: 5873.

Aveni, A.F.; Calnek, E. y Hartung, H. (1988): «Myth, environment, and the orientation of the Templo Mayor of Tenochtitlan". American Antiquity, 53: 287-304.

AVENI, A.F. y URTON, G. (eds.) (1982): “Ethnoastronomy and Archaeoastronomy in the American Tropics". Annals of the New York Academy of Sciences 385. The New York Academy of Sciences. New York.

BAITY, E.C. (1969): «Some Implications of Astro-Archaeology for Americanists». Verhandlungen des 38 Internationalen Amerikanistenkongresses, 1: 85-94, Klaus Renner. München.

- (1973): «Archaeoastronomy and Ethnoastronomy so far». Current Anthropology, 14: 389-449.

BARLAI, K. (1980): «On orientation of graves in prehistoric cemeteries". Archaeoastronomy (JCA), 3, 4: 29-30.

Baschmakoff, A. (1930): «Les alignements de Carnac (Morbihan)». L'anthropologie, 40: 40-75.

BEACH, A.D. (1977): «Stonehenge I and lunar dynamics». Nature, 265, 5589: 17-21.

BENSON, A. y Hoskinson, T. (eds.) (1985): "Earth and Sky: Papers from the Northridge Conference on Archaeoastronomy". Slo'w Press. Thousand Oaks.

Berthelot, R. (1949): “La pensée de l'Asie et l'astrobiologie". Payot. Paris.

BRANDT, J.C.; MARAN, S.P.; WilliamSON, R.A.; HARRINGTON, R.S.; COCHRAN, C.; KENNEDY, M.; KENNEDY, W.J. y VON DEL ChAMBERLAIN (1975): «Possible rock art records of the Crab Nebula supernova in the Western United States». En A.F. Aveni (ed.): 45-58. 
Brandt. J.C. y WII.l.IAMSON, R.A. (1977): «Rock Art Representations of the AD. Supernova: A Progress Reportn. En A.F. Aveni (ed.): 171-177.

- (1979): "The 1054 Supernova and Native American Rock Art». Archaeoastronomy (JHA), 1: S1-S38.

BRECHER, K. (1977): "Sirius Enigmas". Technology Review, 80, 2: 53-63.

BRINCKERHOFF, R.F. (1976): «Astronomically-oriented markings on Stonehengem. Nature, 263, 5577: 465-469.

BRODA, J. (1978): «Cosmovisión y estructuras de poder en el México prehispánicon. Comunicaciones Proyecto Puebla-Tlaxcala. 15: 165-172.

- (1982): “Astronomy. Cosmovisión, and Ideaology in Pre-Hispanic Mesoamericam. En A.F. Aveni y G. Urton (eds.): 81-110.

- (1991): «The Sacred Landscape of Aztec Calendar Festivals: Myth, Nature, and Societym. En D. Carrasco (ed.): "To Change Place. Aztec Ceremonial Landscapes". University Press of Colorado, Niwot: 74-120.

Broda, J; IWANISZEWSKI, S. y MAUPOME, L. (eds.) (1991): "Arqueoastronomía y etnoastronomía en Mesoamé. rica". Universidad Nacional Autónoma de México. México D.F.

BURL, A. (1976): "The stone circles of the British Isles". Yale University Press. New Haven.

- (1979)a: "Prehistoric Avebury". Yale University Press. New Haven.

- (1979)b: "The prehistoric stone circles of Britain and Ireland". Frances Lincoln. London.

- (1980): «Science or symbolism: problems of archaeoastronomy». Antiquity, 54, 212: 191-200.

- (1983): "Prehistoric Astronomy and Ritual". Shire Archaeology. Aylesbury.

- (1987): "The Stonehenge people". J.M. Dent \& Sons. London.

CARLSON, J.B. (1979): «Archaeoastronomy - the scope and implications in interaction with other disciplines". $\mathrm{Ar}$ chaeoastronomy (BCA), 2, 2: 7-9.

CARLSON, J.B. y JUdGE, W.J. (eds.): (1987): “Astronomy and Ceremony in the Prehistoric Soutwest". Papers of the Maxwell Museum of Anthropology no. 2. University of New Mexico. Albuquerque.

CARrasco, D. (1989): «The king, the capital and the stars: the symbolism of authority in Aztec religion». En A. F. Aveni (ed.): 45-54.

Chamberlain, Von Del (1982): "When the Stars Came Down to Earth. Cosmology of the Skidi Pawnee Indians of North America". Ballena Press y Center for Archaeoastronomy. Los Altos.

Charriere, G. y Roos, A. (1964): «Stonehenge, temple votif et monument calendaire». Bulletin de la Société Préhistorique Française, 61, 1: 169-184.

Clark, D. H. y Stevenson, F.R. (1977): "The Historical Supernovae". Pergamon Press. Oxford.

Colton R. y Martin, R.L. (1967): «Eclipse Cycles and Eclipses at Stonehengen. Nature, 213: 476-478.

DeARBorn, D.S.P. y SCHREIBER, K.J. (1986): «Here Comes the Sun: The Cuzco-Machu Picchu Connection». Archaeoastronomy (JCA), 9, 1-4: 15-37.
Dow. J.W. (1967): "Astronomical orientations at Teotihuacan: A Case Study in Astro-Archaeologyn. American Antiquity. 32, 3: 326-334.

EdDY, J.A. (1981): "Some Thoughts on Archaeoastronomy Todayn. En R.A. Williamson (ed.): 21-24.

EliAdE, M. (1973): "Lo sagrado y lo profano". Guadarrama. Madrid.

EllegARD, A. (1981): "Stone Age Science in Britain?». Current Anthropology, 22, 2: 99-125.

FARRER, C. (1990): «"Blue Archaeoastronomy": Ethnoastronomy, 1983-1989" Ponencia presentada al Third "Oxford" International Symposium on Archaeoastronomy.

FARRER, C.R. y SECOND, B. (1981): "Living the sky: aspects of Mescalero Apache ethnoastronomy». En R. A. Williamson (ed.): 137-150.

FARRER, C.R. y WILlIAMSON, R.A. (1991): «Epilogue: blue archaeoastronomy». En R.A. Williamson y C.R. Farrer (eds.): "Earth and sky: visions of the cosmos in native American folklore". University of New Mexico Press. Albuquerque.

Firneis, M.G. y LADENBaUeR-Orel, H. (1978)a: «Studien zur Orientierung der Kirchen von St. Ulrich in Wieselburg und St. Ruprecht in Wien». Forschungsberichte zur Ur- unf Frühgeschichte, 10: 124-126.

- (1978)b: «Studien zur Orientierung der Kirchen con St. Ulrich in Wieselburg und St. Ruprecht in Wien». Forschungsberichte zur Ur- und Frühgeschichte, 23: 1-14.

FLeming, A. (1975): «Megalithic astronomy: a prehistorian's view". Nature, 255, 5510: 575.

Freeman, P.R. (1982): «The statistical approach». En D. C. Heggie (ed.): 45-52.

Freeman, P.R. y Elmore, W. (1979): «A test for the significance of astronomical alignments". Archaeoastronomy (JHA), 1: S86-S96.

GiBBS, S.L. (1979): «Archaeoastronomy and the history of astronomy». Archaeoastronomy (BCA), 2, 2: 9-10.

GiNGERICH, O. (1989): «Reflections on the role of archaeoastronomy in the history of astronomym. En A.F. Aveni (ed.): 38-44.

Guzsıx, T. (1978): «Sol aequinoctialis. Zur Frage de äquinoktialen Ostung in Mittelalter». Periodica Politechnica - Architecture, 22: 191-213.

Hawkes, J. (1967): "God in the Machine». Antiquity, 41: 174-180.

Hawkins, G.S. (1963): "Stonehenge decoded". Nature, 200: 306-308.

- (1964): «Stonehenge: a neolithic computer». Nature, 202: $1258-1261$.

- (1965): "Callanish, a Scottish Stonehenge». Science, 174, 3654: 127-130.

- (1968): «Astro-Archaeology». Vistas in Astronomy, 10: 45-88.

Hawkins, G.S. y White, J.B. (1965): "Stonehenge decoded". Doubleday. New York.

Hedges, K. (1981): «Winter Solstice Observatory Sites in Kumeyaay Territory, San Diego County, California». En R.A. Williamson (ed.): 151-156.

- (1987): «Methodology and Validity in California Archaeoastronomy". En A. Benson y T. Hoskinson (eds.): $25-40$. 


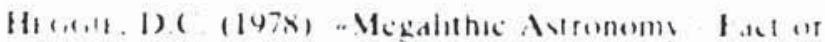

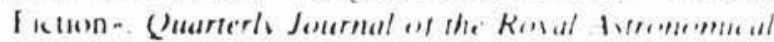

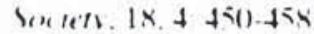

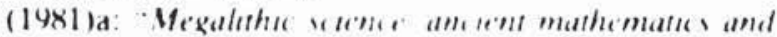
astronems in verehern turepe" Ihames \& Hudon London.

- (1981)h: alighlight and prohlems of megalithic astro.

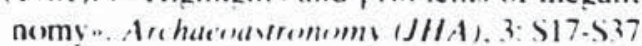

- (1962)at (cd) "Archaecastronomv in the ()ld Wiorld" ( ambridge I nucrsty Press. ( ambridge.

(14x:)h. Mcgalithic astronomy: highlights and pro. blems.. In D)( Heggic (c.d.) 1-24

H(:) Anctent World. Philonophical Iransactoms of the Roval Socicts of London A. 276. 1257.

Horil. F. (IYho) "Stonchenge - an celipse predictor" Nature. 211: 4.54-4.56.

- (1966)b: "Speculations on Stonchenge". Antiquity. $\mathcal{H})$ : $262-276$.

Hidson. T:: L.h . (i. y HoDxifs, K. (1979): *Solstice Observers and Observatories in Native California". Journal of California and Great Basin Anthropology, 1. 1: 39-63.

HUDSON. T. y UNDF RHAy. F. (1978): " Crustals in the Skv: An Intellectual Odvise' Involving (humash Astronomv, (osmolegv and Rock Ari". Ballena Press.

IWANIS/E WSKI. S. (1989): "Exploring some anthropological theoretical foundations for archaeoastronomyn. En A. F. Aveni (ed.): 27-37.

- (1990): “Astronomiia kak kulturnaia sistema". En A. A. Gurshtein (ed.): “Na rubezhakh poznaniia vselennov". Nauka. Moskva: 67-73.

- (1991)a: «Astronomy as a Cultural System». Interdisciplinarni izsledvaniya, 18: 282-288.

- (1991)b: «III Miedzynarodowa Oksfordzka Konferencja Archeoastronomiczna w St. Andrews". Kurartalnik Historii Vauki i Te'chniki. 36. 2: 166-169.

- (1992)a (ed.): "Readings in Archaeoastronomy". State Archaeological Museum. Department of Historical Anthropology, Institute of Archaeology. Warsaw University. Warsaw.

- (1992)b: "Archaeoastronomy and Ethnoastronomy in Poland: the Last Two decades". En S. Iwaniszewski (ed.): $57-70$.

- (1993): "Mesoamerican cross-circle revisited". En C.L. N. Ruggles (ed.): 288-297.

KEHOE, A.B. (1981): "The Cultural Significance of the Moose Mountain Observatory". Archaeoastronomy (JHA), 4, 1: 8-9.

Kendall, D.G. (1974): «Hunting quanta». En F.R. Hodson (ed.): 231-266.

KOBERL, C. (1984): "On the astronomical orientation of St. Vitus' Cathedral and St. George's Church in the Castle of Prague". Bulletin of the Astronomical Institutes of Czechoslovakia, 35: 216-220.

KOHLER, U. (1991): "Pittfalls in archaeoastronomy: with examples from Mesoamerica». Rivista di Archeologia, supplementi, 9: 130-136.

KRUPP, E.C. (1981): "A glance into the smoking mirror". En R.A. Williamson (ed.): 55-59.
1 lost) (cd): "Archaceastronoms and the roos of "rence" A A AS Selected Simposium no. 71. Westben Prew Buslder

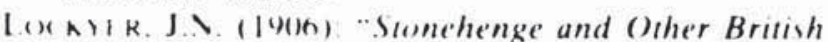
Stome Menuments Astronomically considered". Macmillan. Landon.

Mar KiH. F. (1974): "Archacological tests on supposed prehistoric astronomical sites in Scotland". En F.R. Hexison (ced.): $169-194$.

(1977)a: "Sctence and societs in prehistoric Britain". Paul Elck. London.

(1977)h: "The' me'galith builders". Phaidon. Oxford.

(1981): "The last word on archacoastronomy?" Archaeosstronomi B(A). 4. 1:6.

(14א8): "Investigating the prehistoric solar calendar". I:n (.I... Ruggles (cd.): "Records in stone: papers in memory of Alexander Thom". Cambridge Liniversity Press. (ambridge: 2(k-231.

Marsha(k. A. (1964): "Lunar Notation on Upper Paleolithic Remains". Science, 146. 364.5: 743-745.

- (1972): "The Roots of Civilization". McGraw-Hill. New York.

MCCluskeY. S.C. (1981): "Transformations of the Hopi (alendar". En R.A. Williamson (ed.): 173-182.

(1982): "Archacoastronomy. Ethnoastronomy and the History of Science". En A.F. Aveni y G. Urton (eds.): 34.3-3.5i

- (1989): “Mid-yuarter days and the historical survival of British folk astronomy". Archaeoastronomy (JHA), 13: S1-S19.

MAYER, D. (1979): “Miller's Hypothesis: Some California and Nevada Evidencen. Archaeoastronomy (JHA), 1: S51-S74.

MIDGLEY, M.S. (1985): "The origin and function of the earthen long barrows of Northern Europe". British Archaeological Reports. International Series 259. Oxford.

Milı. W. W.C. (1955)a: “Two prehistoric drawings of possible astronomical significance». Astronomical Society for the Pacific, Leaflet 314.

- (1955)b: «Two possible astronomical pictographs found in Northern Arizona". Plateau, 27, 4: 6-13.

Moir, G.; RugGles, C.L.N. y Norris, R. (1980): «Megalithic science and some Scottish site plans". Antiquity, 54, 210: 37-200.

Moreno Corral., M. (ed.) (1983): "Historia de la astronomia en México". Universidad Nacional Autónoma de México. México D.F.

NEWHAM, C.A. (1966): "Stonehenge - a neolithic "observatory" ". Nature, 211: 456-458.

Pasztor, E. (1992): «Archaeoastronomical Research and Problems in Hungary». En S. Iwaniszewski (ed.): 2024.

PATRICK, J. (1979): "A reassessment of the lunar observatory hypothesis for the Kilmartin Stones». Archaeoastronomy (JHA), 1: S78-S85.

Pleslova-ŚTikova, E. (1990): «Umfriedungen und befestigte Siedlungen aus dem Äneolithikum Böhmen. Versuch einer kulturhistorischen Interpretation». Jahresschrifte für mitteldeutsche Vorgeschichte, 73: 191-201. 
PIfSIONA.ŚtIRONA. E.: MARFK. F. y HORSKY. Z. (1980): "A Square Enclosure of the Funnel Beaker Culture (350) B.( :): at Makotfasy (central Bohemia): A paleoastronomic Structure". Archeologicke rozhledy. 32: 335. 119-120.

Radosi avova. Ts. (1993): "Astronomical knowledge in Bulgarian lands during the Neolithic and Early Bronze Age. En C.L.N. Ruggles (ed.): 107-116.

RAPPAPORT, R.A. (1971)a: "Ritual, sanctity, and cybernetics». American Anthropologist, 73:59-76.

- (1971)b: "The sacred in human evolution". Annual Review of Ecology and Systematics. 2:23-46.

RFYmaN. J. (1976): "Archaeology, astronomy, and adaptation at Pueblo Boniton. Science, 196.4257:957-962.

- (1979): "Some observations on archaeology and archaeoastronomym. Archaeoastronomy (BCA). 2.2:11-13.

- (1980): "The Predictive Dimension of Priestly Power". En C.L. Riley y B.C. Hendrick (eds.): "New Frontiers in the Archaeology and Ethnohistory of the Greater Southwest". Transactions of the Illinois Academy of Science, 74, 4: 40-59.

Robinson, J.H. (1970): "Sunrise and Moonrise at Stonehengen. Nature, 225, 5239: 1236-1237.

Romano, G. (1992): "Archeoastronomia italiana". Cooperativa Libraria Editrice Università di Padova. Padova.

RoY, B.C. (1987): "Stonehenge: A New Theory". History of Religions, 26, 3: 225-278.

RugGles, C.L.N. (1981): «A critical examination of the megalithic lunar observatoriesn. En CLN. Ruggles y A.W.R. Whittle (eds.): 153-209.

- (1982)a: «Megalithic astronomical sightlines: current reassesment and future directions». En D.C. Heggie (ed.): 83-105.

- (1982)b: «A reassesment of the high precision megalithic lunar sightlines, 1: Backsights, indicators and the archaeological status of the sightlines". Archaeoastronomy (JHA), 4: S21-S40.

- (1983): «A reassesment of the high precision megalithic lunar sightlines, 2: foresights and the problem of selection». Archaeoastronomy (JHA), 5: S1-S36.

- (1984)a: "Megalithic astronomy: a new archaeological and statistical study of 300 Western Scottish sites". With contributions by P.N. Appleton, S.F. Burch, J.A. Cooke, R.W. Few, J.G. Morgan y R.P. Norris. British Archaeological Reports, British Series 123. Oxford.

- (1984)b: "Megalithic astronomy: the last five years". Vistas in Astronomy, 27: 231-289.

- (1990): «A statistical examination of the radial line azimuths at Nazca». En A.F. Aveni (ed.): 245-269.

- (1993) (ed.): "Archaeoastronomy in the 1990s". Group D Publications. Loughborough.

- n.d.a.: «The role of excavation in archaeoastronomy". (Székesféhervar, 1991). Bulletin of the Institute of Astronomy, Hungarian Academy of Sciences. Budapest (en prensa).

RugGles, C.L.N.; MEdYCKYJ-SCOTT, D.J. y GRUFFyd, A. (1993): «Multiple viewshed analysis using GIS and its archaeological application: a case study in northern Mull». En J. Andersen, T. Madsen y I. Scollar (eds.): "Computer Applications and Quantitative Methods in
Archaeology 1992 (CAA 92)". University of Arhus Pres. Arhus:125-132

Ri colls. ( L.N. y SAl NDERS. N.J. (e.p.): "The study of cultural astronomy.. Ponencia presentada a la Conferencia Internacional sobre Arqueoastronomía "Oxford 3". 1490. En ( I..N. Ruggles y N.J. Saunders (eds.): "Astronomies and (ultures". University Press of Colorado. Niwot. Colorado.

Riggl.fs, C.L.N. y WhitTl. A.W.R. (eds.): (1981): “Astronomy and societs in Britain during the period $4(N)$. 150) $B C^{*} \cdot$. BAR International Series 88 . Oxford.

SADOUSKI. R.M. (1989): "A few remarks on the astronomy of R.T. Zuidema's quipu calendar”. En M.S. Ziólkowski y R.M. Sadowski (eds.): 209-213.

S(HAEFtR. B.E. (1986): "Atmospheric extinction effects on stellar alignments». Archaeoastronomy (JHA), 10: S32-S42.

SCHI.OSSER. W. y CiERnY, J. (1982): “Astronomical Orientation of Neolithic Sites in Central Europe». En D.C. Heggie (ed.): 225-230.

SpraJC, I. (1987-88): «Venus and Temple 22 at Copan: Revisited". Archaeoastronomy (JCA), 10: 88-97.

- (1993): "Venus orientations in ancient Mesoamerican architecture». En C.L.N. Ruggles (ed.): 270-277.

TFDLock. B. (1982): "Time and the Highland Maya". University of New Mexico Press. Albuquerque.

Tном, A. (1954): "The solar observations of megalithic man". Journal of the British Astronomical Association, 64, 8: 396-404.

- (1955): "A statistical examination of the megalithic sites in Britain". Journal of the Royal Statistical Society, A. III, 118: 275-296.

- (1961): «The egg-shaped standing stone rings of Britain". Archives Internationales d'Histoire des Sciences, 14, 56-57: 291-303.

- (1966)a: «Megalithic astronomy: indications in standing stones». Vistas in Astronomy, 7: 1-57.

- (1966)b: «Megaliths and Mathematics». Antiquity, 40: 121-128.

- (1967): “Megalithic sites in Britain". Oxford University Press. London.

- (1971): "Megalithic lunar observatories". Oxford University Press. Oxford.

- (1974): "Astronomical significance of prehistoric monuments in Western Europe». En F.R. Hodson (ed.): 149-156.

Тном, А. у Тном, A.S. (1972)a: «The Carnac alignments". Journal for the History of Astronomy, 3, 1: 1126.

- (1972)b: «The uses of alignments at La Menec, Carnacm. Journal for the History of Astronomy, 3: 151-164.

- (1973): "The Kerlescan cromlechs". Journal for the History of Astronomy, 4: 168-173.

- (1974): "The Kermario alignments". Journal for the History of Astronomy, 5, 1: 30-47.

- (1978): "Megalithic remains in Britain and Brittany". Oxford University Press. Oxford.

- (1980): "Astronomical foresights used by megalithic man». Archaeoastronomy (JHA), 2: S90-S94.

- (1982): «Statistical and philosophical arguments for the astronomical significance of standing stones with a se- 
lection on the solar calendar.. Fn D. Hegge led) 53-82.

-. (1983): "Observation of the M(x)n in megalithic times" Archaeoastronomv. (JHA). 5 \$57-. Soxh.

Тном, A.: Тном, A.S. у Тном, A.S. (1974): «Stonehenge . Journal for the Historv of Astronomy. 5: 71-(x).

- (1975): "Stonchenge as a possibly lunar observatory". Journal for the Historv of Astronomy, 6. 1: 19-30.

THOM. A.: TH()M. A.S. y BURL, A. (1980): "Megalithic Rings. Plans and Data for 229 Monuments in Britain". Britısh Archaeological Reports. British Series 81. Oxford.

IHOM. A.S.: KFR, J.M.D. y BIRROWS, T.R. (1988): “The Bush Barrow gold lozenge: is it a solar and lunar ca. lendar for Stonehengen. Antiquity. 62: 492.5()2.

URION. G. (1981): "At the Crowsoads of the Earth and the Sky. An Andean Cosmology.. University of Texas Press. Austin.

Wheatley. P. (1971): "Pivot of the Four Quarters". Aldine Press. Chicago.

Williamson, R.A. (ed.) (1981): "Archaeoastronomy in the Americas". Ballena Press y Center for Archaeoastronomy. Los Altos.

WILl.IAMSON, R.A.; FISHER, H.J. y O'FLYNN, D. (1977): "Anasazi Solar Observatoriesn. En A.F. Aveni (ed.): 203-217

WOOD. J.E. (1978): "Sun, moon and standing stones". Oxford University Press. Oxford.

Young, M.J. y Williamson, R.A. (1981): “Ethnoastronomy: The Zuni Casen. En R.A. Williamson (ed.): 183-191.
Yin vi. J. (1979): "Seminar summary: in search of archaeoastronomy: what is it and where is it going". Archaeoastronomv (BC A). 2, 2: 14-15.

ZF II IK. M. (198.3): "Onc Approach to Archaeoastronomy: An Astronomer's Viewn. Archaeoastronomy (JCA), 6. 1.4: 4.7.

- (1984)a: *The Ethnoastronomy of the Historic Pueblos. I: Calendrical Sun Watching". Archaeoastronomy, (JHA), 8: S1-S24.

- (1984)b: “Summer Solstice at Casa Rinconada: Calendar. Hierophany, or Nothing?" Archaeoastronomy (JCA), 7. 1-4: 76-81.

- (1985)a: "Sun Shrines and Sun Symbols in the U.S. Southwest ". Archaeoastronomy, (JHA), 9: S86-S96.

- (1985)b: "The Fajada Butte Solar Marker: A Reevaluatuon.. Science, 228, 4705: 1311-1313.

- (1985)c: "A Reassesment of the Fajada Butte Solar Markern. Archaeoastronomy (JHA), 9: S69-S86.

- (1986): "The Ethnoastronomy of the Historic Pueblos. II: Moon Watching". Archaeoastronomy (JHA), 10: S1-S22.

Zıłkowskı, M.S. (1989): «Knots and kinks. The quipu-calendar or supposed Cuzco luni-solar calendar». En M. Ziołkowski y R. M. Sadowski (eds.): 197-208.

Zıoł.Kowskı. M.S. y LeBEUf, A. (1993): «Were the Incas able to predict lunar eclipses?». En C.L.N. Ruggles (ed.): 298-308

Zıю.kowski, M.S. y SADOwSKI, R.M. (eds.) (1989): “Time and Calendars in the Inca Empire". BAR International Series 479, Oxford. 


\section{TABLA 1. CAMBIOS CONCEPTUALES EN LOS ESTI DIOS SOBRF ACTIVIDADES ASTRONOMICAS Y (CAIENDARICAS:}

astroarqueología

astroarqueología

astroarqueología

astroarqueología

astroetnología

astronomía megalítica, ciencia megalítica, ciencia de la Edad de Piedra

arqueología estelar (star archaeology)

arqueoastronomía

arqueoastronomía

arqueoastronomía

arqueoastronomía

arqueoastronomía/ etnoastronomía

arqueoastrofísica

astronomía cultural estudio de orientaciones de monumentos megaliticos. auxiliar de la arqueología, antropología, historia. etc. (Hawkins y White. 1965: 121)

parte de la historia de la ciencia (de la astronomía) (Hawkins. 1968: 45)

subdisciplina antropológica. técnica para estudiar las ciencias exactas en la antigüedad (Baity, 1969: 85)

subdisciplina de la arqueoastronomía, referente a las ciencias exactas: el trabajo de campo (mediciones): y los cálculos posteriores (Aveni, 1981b: 1)

investigación paralela a la astroarqueología de Hawkins, referente a los estudios en las Américas (Baity, 1969: 85)

tratamiento del conocimiento astronómico de los constructores de monumentos megalíticos como un saber científico (Thom, 1966, 1967: 92-106)

sinónimo de la astroarqueologia, atribuido por MacKie (1981: 6) a Hawkins

"estudio de las prácticas astronómicas en tiempos antiguos". el término introducido por MacKie en 1971 (MacKie. 1981: 6)

subdisciplina particular. complementaria de la etnoastronomía (Baity, 1973: 389-390, 422)

interdisciplina que resulta de la cooperación entre investigadores procedentes de las ciencias exactas (astrónomos, sobre todo): y de las humanidades (arqueólogos, antropólogos, etnólogos, etc.) (Aveni, 1975: xiv y trabajos posteriores)

parte de la historia de la astronomía (Gibbs, 1979)

parte de la historia de la ciencia que estudia también el contexto social de la ciencia (McCluskey, 1982: 350)

enfoque particular de la arqueoastronomía orientado a contribuir al desarrollo de la ciencia moderna (novas, supernovas, cometas, colores de estrellas) (Brecher, 1977)

el estudio de las relaciones entre el hombre y los fenómenos astronómicos dentro del contexto cultural; se compone de 4 subdisciplinas: la arqueoastronomía, la etnoastronomía, la historia de la astronomía y la socioastronomía (Iwaniszewski, 1990, 1991) 
TABIA 2 SIMPOSIUS ARUIEOUASIRUNOMICOS MAS IMPORTANTES.

197375-1981:

- 1980. San Francisco. EE.UU; “Arqueoastronomía y las raices de la Ciencia” por la Asociación Americana para el Progreso de la Ciencia (Krupp. 1984).

- 1980). Universidad de Newcastle-upon-Tyne. Inglaterra (Ruggles y Whittle. 1981).

- 1981. Nueva York. EE.UU: "Etnoastronomía y Arquecoastronomía en los Trópicos Americanos" por la Academia de Ciencias de Nueva York (Aveni y Urton. 1982).

\section{1-1986:}

- 1982. Ensenada. México: "Historia de la astronomía en México" (Moreno Corral. 1983).

- 1982. Washington. D.C.. EE.UU: "Cosmología africana. astronomía y cosmovisión”. organizado por la Asociación de Estudios Africanos.

- 1983. Washington. D.C.. EE.L L Primer Simposio Internacional sobre Etnoastronomía.

- 1983. Alhuquerque, EE.UU: “Astronomia y Circmonia en el SurOeste Prehistórico” (Carlson y Judge, 1987).

- 1983. Northridge. EE.UU: "Primera Conferencia Regional Occidental sobre Arqueoastronomía" (Benson y Hoskinson. 1985).

- 1984. México D.F.. México; “Arqueoastronomía y Etnoastronomía en Mesoamérica” (Broda et alii, 1991 ).

- 1984. Little Rock. EE.UU: “Arte Rupestre y Arqueoastronomía”.

- 1985. Padova, Italia; "Investigación arqueoastronómica en Italia”.

\section{6-1990:}

- 1988. Tolbukhin (hoy Dobrikh). Bulgaria: Primer Simposio Nacional sobre Arqueoastronomía (Interdisciplinarni izsledvaniya 17,18 ).

- 1989. Venecia. Italia: “Astronomia c Archeologia” (Rivista di Archeologia, Supplementi, 9).

\section{$1990-1993$}

- 1990. Varsovia, Polonia: "Problemas actuales y el futuro de la arqueoastronomía" (Iwaniszewski, 1992).

- 1991, Székesfehérvar. Hungría: "Problemas actuales y el futuro de la arqueoastronomía".

- 1992, Frombork, Polonia; "El Tiempo y la Astronomía en el Encuentro de Dos Mundos".

- 1992. Guatemala-Tikal, Guatemala; "Conferencia pan-americana sobre las matemáticas, astronomía y modos de pensar precolombinos".

- 1992, Estrasburgo, Francia: Conferencia Europea sobre Arqueoastronomía.

- 1993, Smolyan. Bulgaria: $4^{\mathrm{A}}$ Conferencia Europea sobre Arqueoastronomía. 